\title{
A strategic approach to estate division problems with non-homogenous preferences
}

Citation for published version (APA):

Palvolgyi, D. G., Peters, H. J. M., \& Vermeulen, A. J. (2010). A strategic approach to estate division problems with non-homogenous preferences. METEOR, Maastricht University School of Business and Economics. METEOR Research Memorandum No. 036 https://doi.org/10.26481/umamet.2010036

Document status and date:

Published: 01/01/2010

DOI:

10.26481/umamet.2010036

Document Version:

Publisher's PDF, also known as Version of record

\section{Please check the document version of this publication:}

- A submitted manuscript is the version of the article upon submission and before peer-review. There can be important differences between the submitted version and the official published version of record.

People interested in the research are advised to contact the author for the final version of the publication, or visit the DOI to the publisher's website.

- The final author version and the galley proof are versions of the publication after peer review.

- The final published version features the final layout of the paper including the volume, issue and page numbers.

Link to publication

\footnotetext{
General rights rights.

- You may freely distribute the URL identifying the publication in the public portal. please follow below link for the End User Agreement:

www.umlib.nl/taverne-license

Take down policy

If you believe that this document breaches copyright please contact us at:

repository@maastrichtuniversity.nl

providing details and we will investigate your claim.
}

Copyright and moral rights for the publications made accessible in the public portal are retained by the authors and/or other copyright owners and it is a condition of accessing publications that users recognise and abide by the legal requirements associated with these

- Users may download and print one copy of any publication from the public portal for the purpose of private study or research.

- You may not further distribute the material or use it for any profit-making activity or commercial gain

If the publication is distributed under the terms of Article $25 \mathrm{fa}$ of the Dutch Copyright Act, indicated by the "Taverne" license above, 
Dénes Pálvölgyi, Hans Peters, Dries Vermeulen

A strategic approach to estate division problems with non-homogenous preferences

$\mathrm{RM} / 10 / 036$

\section{METEOR}

Maastricht University School of Business and Economics

Maastricht Research School of Economics

of Technology and Organization

\section{PO. Box 616}

NL - 6200 MD Maastricht

The Netherlands 


\title{
A strategic approach to estate division problems with non-homogenous preferences
}

\author{
Dénes PÁlvölgyi Hans Peters \\ Dries Vermeulen *
}

July 2010

\begin{abstract}
The classical bankruptcy problem (O'Neill, 1982) is extended by assuming that the agents have non-homogenous preferences over several estates. A special case is the one in which there are finitely many estates and the agents have homogenous preferences, i.e., constant utilities, per estate. In the general case, i.e., the infinite estate problem, players have arbitrary preferences over an interval of real numbers each of which is regarded as an estate. A strategic game is formulated in which each agent/player distributes his legal entitlement over the estates, resulting in individual claims per estate: each estate is then divided proportionally according to these individual claims. The focus of the paper is on the study of Nash equilibria, in particular on their existence, in finite and infinite estate games. It is also shown that, generally speaking, Nash equilibria are not unique nor Pareto optimal but that they are Pareto optimal in a second best sense: they do not Pareto dominate each other. The paper concludes with a brief consideration of envy-freeness.
\end{abstract}

JEL Classification numbers: C72, D63

Keywords: Bankruptcy problem, estate division, non-homogenous preferences

\section{Introduction}

The classical bankruptcy problem (O'Neill, 1982; Aumann and Maschler, 1985) is the problem of dividing an estate - for instance a heritage if someone has died, or the leftovers from a bankrupt firm - among a group of claimants who have legal entitlements to the estate - for instance family members, debt holders, share holders. This problem has been approached in a normative, axiomatic

* Department of Quantitative Economics, Maastricht University, P.O. Box 616, 6200 MD Maastricht, The Netherlands. Email addresses: d.palvolgyi@maastrichtuniversity.nl, h.peters@maastrichtuniversity.nl, d.vermeulen@maastrichtuniversity.nl. 
way: see Thomson (2003) for an overview of such approaches. Starting with O'Neill (1982) and extended by Atlamaz et al. (2008), the problem has also been approached strategically, by formulating a non-cooperative game associated with the bankruptcy problem and investigating its Nash equilibria.

In the original bankruptcy problem preferences are assumed to be homogenous: basically, the estate is an amount of money and each agent wants to maximize his allocation. In the present paper preferences can be non-homogenous. We model this by assuming that there is a finite or infinite number of estates: per estate each agent's preference is homogenous, expressed by a constant utility value, but across estates an agent's preference may vary. In the case of infinitely many estates, we assume that each estate is a point in the interval $[0,1]$, and an agent's preference is represented by a utility function defined on this interval. In a typical example, the estate is a (one-dimensional) piece of land, and agents do not only care about the amount of land they obtain but also about the quality, where different agents may value pieces of land differently. The non-cooperative game we consider is similar to the one already proposed by O'Neill (1982) and Atlamaz et al. (2008). Each agent distributes his legal entitlement over the estates, and each estate is divided proportionally to the individual claims on it. The game bears resemblance to certain procedures proposed in the fair division literature, see for instance Brams and Taylor (1996).

Throughout the paper we use the estate division terminology, but the model has applications other than the division of an heritage or the leftovers of a bankrupt firm. For instance, one may think of the interval $[0,1]$ as a continuum of uniformly distributed consumers (cf. Hotelling, 1929), and of the claimants as firms who provide services to these consumers, with each firm providing total service equal to its entitlement. In this case, each part of the consumer continuum is distributed proportionally with respect to the claims - now called investments - of the firms. Note that this interpretation naturally allows for competitive investments by different firms in one and the same consumer segment, thus, for multiple claims in our model.

Other applications concern political problems, auctions, or problems of land division. The shares of the players may be interpreted as probabilities of winning in political elections (Merolla, Munger, and Tofias, 2003) or auctions (Cramton, Gibbons, and Klemperer, 1987). Land division has already been mentioned above; see Berliant (1985) and Berliant et al. (1992), among others. In the model with finitely many estates another potential application may arise by regarding the estates as finitely many roads from A to B. The agents are transportation companies who distribute their trucks over different roads, and the road capacity is shared proportionally to the numbers of trucks: in this application, though, it would be more realistic to let the utility of an estate, viz. the use of a road, depend on the total claim put on it. See Gairing et al. (2009).

The main part of this paper is devoted to characterizing and establishing existence of Nash equilibria. We first prove the existence of so-called $\varepsilon$-equilibria in the model with finitely many estates by a fixed point argument. In an $\varepsilon$ equilibrium each player is obliged to put a claim of minimally $\varepsilon$ on each estate. Existence of Nash equilibria (i.e., $\varepsilon=0$ ) is then obtained by a limit argument. A 
direct proof of this would be complicated by the fact that best replies need not exist. Next, we introduce the infinite estate model and show existence of Nash equilibrium by a limit argument using the finite estate game. Finally, we present an example in which Nash equilibrium does not have to be unique, but identify a special class of estate games that do have a unique Nash equilibrium. We also show that, although Nash equilibria are not unique in general, they cannot Pareto dominate each other. We conclude with a consideration of envy-freeness of equilibrium allocations.

\section{The finite estate problem}

\subsection{The finite estate problem and the finite estate game}

A set $M=\{1, \ldots, m\}$ of estates is to be divided among a set $N=\{1, \ldots, n\}$ of agents. Throughout, $m \geqslant 1$ and $n \geqslant 2$. Each agent $i$ has an entitlement $c_{i}>0$, and a utility $u_{i s}>0$ for ownership of the complete estate $s$. We write $u_{i}=\left(u_{i 1}, \ldots, u_{i m}\right)$. The quadruple

$$
(M, N, c, u)
$$

where $u=\left(u_{1}, \ldots, u_{n}\right)$ and $c=\left(c_{1}, \ldots, c_{n}\right)$, is a finite estate problem.

A solution to the finite estate problem is an allocation of shares of the estates to the agents. More precisely, if $x_{i s} \geqslant 0$ is the share of estate $s$ allocated to agent $i$, then $x=\left(\left(x_{i s}\right)_{s \in M}\right)_{i \in N}$ is a solution if $\sum_{i \in N} x_{i s} \leqslant 1$ for all $s \in M$. Agent $i$ 's utility from $x$ is given by $u_{i}(x)=\sum_{s \in M} x_{i s} u_{i s}$. The purpose of this paper is to find solutions as equilibrium outcomes of a non-cooperative game which, naturally, takes the data $(c, u)$ of the problem into consideration.

To this end, with each finite estate problem $(M, N, c, u)$ a finite estate game $(S, \pi)$ is associated in the following way. The players of the game are the agents of the estate problem. A strategy of player $i$ is a vector $\sigma_{i}=\left(\sigma_{i s}\right)_{s \in M}$ of nonnegative numbers, where $\sigma_{i s}$ denotes the claim that player $i$ places on estate $s$, such that

$$
\sum_{s \in M} \sigma_{i s}=m c_{i}
$$

Remark 2.1 To interpret condition (1) one can think of the $m$ estates as pieces of land of equal area, with total area equal to 1 . The number $\sigma_{i s}$ is thought of as the height of player $i$ 's claim on estate $s$. Since the area of estate $s$ is $1 / \mathrm{m}$, player $i$ spends $\sigma_{i s} / m$ of his entitlement $c_{i}$ on estate $s$. This interpretation and the associated condition (1) are convenient when later in the paper the finite model is extended to an infinite model of estate division.

The strategy set of player $i$ is denoted by $S_{i}$, and $S=\prod_{j \in N} S_{j}$ is the set of strategy profiles. The payoff player $i$ receives from estate $s$ at the strategy 
profile $\sigma=\left(\sigma_{j}\right)_{j \in N} \in S$ is

$$
\pi_{i s}(\sigma)=\left\{\begin{aligned}
\frac{\sigma_{i s}}{\sum_{j=1}^{n} \sigma_{j s}} \cdot u_{i s} & \text { if } \sigma_{i s}>0 \\
0 & \text { if } \sigma_{i s}=0 .
\end{aligned}\right.
$$

The total payoff of player $i$ at the strategy profile $\sigma$ is $\pi_{i}(\sigma)=\sum_{s \in M} \pi_{i s}(\sigma)$. Clearly, $\left(\left(x_{i s}\right)_{s \in M}\right)_{i \in N}$ with $x_{i s}=\sigma_{i s} / \sum_{j \in N} \sigma_{j s}$ if $\sigma_{i s}>0$ and $x_{i s}=0$ otherwise for all $s \in M$ and $i \in N$, is a solution to the finite estate problem: in this solution, each estate is divided among the players in proportion to their claims on it.

Definition 2.2 A strategy profile $\sigma$ is a Nash equilibrium in $(S, \pi)$ if for all $i \in N$

$$
\pi_{i}(\sigma) \geq \pi_{i}\left(\sigma \mid \tau_{i}\right)
$$

for all $\tau_{i} \in S_{i}$, where $\left(\sigma \mid \tau_{i}\right)$ is the strategy profile in which player $i$ plays strategy $\tau_{i}$ while all other players $j$ play $\sigma_{j}$.

Notation For a finite estate problem $(M, N, c, u)$ we denote $C=\sum_{i \in N} c_{i}, \underline{c}=$ $\min \left\{c_{i} \mid i \in N\right\}, \bar{c}=\max \left\{c_{i} \mid i \in N\right\}, \underline{u}=\min \left\{u_{i s} \mid i \in N, s \in M\right\}$, and $\bar{u}=\max \left\{u_{i s} \mid i \in N, s \in M\right\}$.

\subsection{Existence of Nash equilibrium in the finite estate game}

Throughout the remainder of this section we consider the finite estate game $(S, \pi)$ associated with the finite estate problem $(M, N, c, u)$. The proof of existence of Nash equilibrium in this game is complicated by the fact that best replies do not always exist. If for some estate $s$ and strategy profile $\sigma \in S$ we have $\sigma_{j s}=0$ for all $j \neq i$, then player $i$ does not have a best reply to $\sigma$ : for $\tau_{i}$ to be a best reply we need $\tau_{i s}>0$ but then player $i$ improves his payoff by decreasing $\tau_{i s}$ (he still obtains all of $s$ since no other player claims it) and increasing his claim on some estate claimed by at least one other player.

In order to circumvent this difficulty we introduce $\varepsilon$-equilibria and show existence of Nash equilibrium by letting $\varepsilon$ go to zero. For any $0 \leqslant \varepsilon<\underline{c}$ let $S_{i}^{\varepsilon}=\left\{\sigma_{i} \in S_{i} \mid \sigma_{i s} \geqslant \varepsilon\right.$ for all $\left.s \in M\right\}$, and $S^{\varepsilon}=\prod_{i \in N} S_{i}^{\varepsilon}$. Then $\sigma \in S^{\varepsilon}$ is an $\varepsilon$-equilibrium in $(S, \pi)$ if $\pi_{i}(\sigma) \geqslant \pi_{i}\left(\sigma \mid \tau_{i}\right)$ for all $i \in N$ and $\tau_{i} \in S_{i}^{\varepsilon}$. Clearly, for $\varepsilon=0$ an $\varepsilon$-equilibrium is a Nash equilibrium.

For a strategy profile $\sigma$, write $T_{s}=\sum_{i \in N} \sigma_{i s}$ and $y_{i s}=\sum_{j \in N \backslash\{i\}} \sigma_{j s}=$ $T_{s}-\sigma_{i s}$. (If no confusion is likely the dependence of these numbers on $\sigma$ is suppressed from notation.) Note that $\sum_{s \in M} T_{s}=m \sum_{i \in N} c_{i}=m C$. The following lemma characterizes $\varepsilon$-equilibria and, for $\varepsilon=0$, Nash equilibria in $(S, \pi)$. A proof can be found in the Appendix.

Lemma 2.3 Let $0 \leqslant \varepsilon<\underline{c}$. The strategy profile $\sigma \in S^{\varepsilon}$ is an $\varepsilon$-equilibrium if and only if $T_{s}>0$ for all $s \in M$ and for every player $i$ there exists $\lambda_{i}>0$ such that

$$
\lambda_{i} \geqslant \frac{y_{i s}}{T_{s}^{2}} \cdot u_{i s}
$$


for all $s \in M$, with equality if $\sigma_{i s}>\varepsilon$.

For each player $i$ the number $\lambda_{i}$ in Lemma 2.3 is the marginal utility of each positively claimed estate $s$ in the strategy profile $\sigma$. Usually we will refer to the numbers $\lambda_{i}$ as the marginal utilities associated with the equilibrium profile $\sigma$. A straightforward consequence of Lemma 2.3 is the following.

Lemma 2.4 Let $0 \leqslant \varepsilon<\underline{c}$. If $\sigma \in S^{\varepsilon}$ is an $\varepsilon$-equilibrium with associated marginal utilities $\lambda_{1}, \ldots, \lambda_{n}$, then for all $i \in N$ and $s \in M$ with $\sigma_{i s}>\varepsilon$ we have $\sigma_{i s}=T_{s}-\lambda_{i} T_{s}^{2} / u_{i s}$.

Our first result is that $\varepsilon$-equilibria exist for positive $\varepsilon$.

Proposition 2.5 Let $0<\varepsilon<\underline{c}$. Then there exists an $\varepsilon$-equilibrium.

Proof. We construct a map $F: S^{\varepsilon} \rightarrow S^{\varepsilon}$, as follows. Let $\sigma \in S^{\varepsilon}$ with associated $T_{s}$ and $y_{i s}$ for $s \in M$ and $i \in N$. For each $i \in N$, define $f_{i}\left(\lambda_{i}\right)=$ $\sum_{s \in M} \max \left\{\varepsilon, T_{s}-\lambda_{i} T_{s}^{2} / u_{i s}\right\}$ for all $\lambda_{i} \geqslant 0$. Then $f_{i}(0) \geqslant \sum_{s \in M} T_{s}=m \sum_{j \in N} c_{j}$ $>m c_{i}$, and $f_{i}$ is strictly decreasing until it reaches its minimum $m \varepsilon<m c_{i}$. Let $\lambda_{i}^{\sigma}$ denote the unique (and positive) value of $\lambda_{i}$ for which $f_{i}\left(\lambda_{i}\right)=m c_{i}$, and define $F(\sigma)_{i s}=\max \left\{\varepsilon, T_{s}-\lambda_{i}^{\sigma} T_{s}^{2} / u_{i s}\right\}$ for all $s \in M$. The map $F$ is continuous and by Brouwer's Theorem has a fixed point $\sigma^{*}$.

Consider player $i$. Then, for all $s \in M, \sigma_{i s}^{*}=F\left(\sigma^{*}\right)_{i s}=\max \left\{\varepsilon, T_{s}^{*}-\right.$ $\left.\lambda_{i}^{\sigma^{*}}\left(T_{s}^{*}\right)^{2} / u_{i s}\right\}$, where $T_{s}^{*}$ is associated with $\sigma^{*}$. Hence, $\sigma_{i s}^{*} \geqslant T_{s}^{*}-\lambda_{i}^{\sigma^{*}}\left(T_{s}^{*}\right)^{2} / u_{i s}$, which implies $\lambda_{i}^{*} \geqslant y_{i s}^{*} u_{i s} /\left(T_{s}^{*}\right)^{2}$ (where $y_{i s}^{*}$ is associated with $\sigma^{*}$ ), with equality if $\sigma_{i s}^{*}>\varepsilon$. Obviously, $T_{s}^{*}>0$ for all $s \in M$. Thus, by Lemma 2.3, $\sigma^{*}$ is an $\varepsilon$-equilibrium.

The proof of Proposition 2.5 would hold for $\varepsilon=0$ as well if one could show that $T_{s}>0$ for all $s$ also in that case. Instead, we will prove existence of a Nash equilibrium by applying a limit argument for $\varepsilon$ approaching 0 . We first establish positive lower and upper bounds on the marginal utilities and the total claims in an $\varepsilon$-equilibrium for all $\varepsilon \geqslant 0$. These bounds do not depend on $\varepsilon$ or on the number of estates $m$. They are used in the existence proof of Nash equilibrium in the finite estate game, and also in the infinite estate game in the next section.

Lemma 2.6 Let $0 \leqslant \varepsilon<\underline{c}$ and let $\sigma \in S^{\varepsilon}$ be an $\varepsilon$-equilibrium with associated marginal utilities $\lambda_{1}, \ldots, \lambda_{n}$ and total claims $T_{1}, \ldots, T_{m}$.

(a) For all $i \in N: \lambda_{i} \leqslant \bar{u} / \underline{c}$.

(b) For all $s \in M: T_{s} \geqslant(n-1) \underline{u} \underline{c} / n \bar{u}$.

(c) For all $s \in M: T_{s} \leqslant 2 C \bar{u} / \underline{u}$.

(d) For all $i \in N: \lambda_{i} \geqslant \underline{u}^{2} \underline{c} / 2 \bar{u} C^{2}$. 
Proof.

(a) Let $i \in N$. There must be an $\hat{s} \in M$ such that $\sigma_{i \hat{s}} \geqslant c_{i}>\varepsilon$ (since $\left.\sum_{s \in M} \sigma_{i s}=m c_{i}\right)$. Then by Lemma 2.3

$$
\lambda_{i}=\frac{y_{i \hat{s}} u_{i \hat{s}}}{T_{\hat{s}}^{2}}=\frac{y_{i \hat{s}}}{T_{\hat{s}}} \cdot \frac{1}{T_{\hat{s}}} \cdot u_{i \hat{s}} \leqslant 1 \cdot \frac{1}{\sigma_{i \hat{s}}} \cdot \bar{u} \leqslant \frac{\bar{u}}{c_{i}} \leqslant \frac{\bar{u}}{\underline{c}} .
$$

(b) Let $s \in M$. Then by Lemma 2.3

$$
\sum_{i \in N} \lambda_{i} \geqslant \sum_{i \in N} \frac{y_{i s} u_{i s}}{\left(T_{s}\right)^{2}} \geqslant \frac{\underline{u} \sum_{i \in N} y_{i s}}{\left(T_{s}\right)^{2}}=\frac{\underline{u}(n-1) T_{s}}{\left(T_{s}\right)^{2}}=\frac{u(n-1)}{T_{s}} .
$$

Hence $T_{s} \geqslant \underline{u}(n-1) / \sum_{i \in N} \lambda_{i} \geqslant(n-1) \underline{u} \underline{c} / n \bar{u}$, where the last inequality follows from part (a).

(c) First, let $r \in M$ and $i, j \in N$ arbitrary. Clearly, $y_{i r}+y_{j r} \geqslant T_{r}$, hence $y_{i r} / T_{r} \geqslant 1 / 2$ or $y_{j r} / T_{r} \geqslant 1 / 2$. Also, by Lemma $2.3, \lambda_{i} \geqslant y_{i r} u_{i r} / T_{r}^{2}$ and $\lambda_{j} \geqslant y_{j r} u_{j r} / T_{r}^{2}$. Hence

$$
\text { for all } r \in M \text { and } i, j \in N: \lambda_{i} \geqslant \underline{u} / 2 T_{r} \text { or } \lambda_{j} \geqslant \underline{u} / 2 T_{r} .
$$

Now take $\hat{r} \in M$ with $T_{\hat{r}} \leqslant C$ (this is possible since $\sum_{s \in M} T_{s}=m C$ ), then by applying (3) with $r=\hat{r}$ we obtain

$$
\text { for all } i, j \in N: \lambda_{i} \geqslant \underline{u} / 2 C \text { or } \lambda_{j} \geqslant \underline{u} / 2 C \text {. }
$$

Let $s \in M$. If $T_{s} \leqslant(n-1) \underline{c}+\bar{c}$ then $T_{s} \leqslant 2 C \bar{u} / \underline{u}$ and we are done. Otherwise, $T_{s}>(n-1) \underline{c}+\bar{c}$ and so there must be at least two players, say $i$ and $j$, with $\sigma_{i s}>\varepsilon$ and $\sigma_{j s}>\varepsilon$. Then by Lemma $2.3, \lambda_{i}=y_{i s} u_{i s} / T_{s}^{2} \leqslant \bar{u} / T_{s}$ and $\lambda_{j}=y_{j s} u_{j s} / T_{s}^{2} \leqslant \bar{u} / T_{s}$. By (4) this implies $\bar{u} / T_{s} \geqslant \underline{u} / 2 C$, hence $T_{s} \leqslant 2 C \bar{u} / \underline{u}$.

(d) Let $i \in N$. There is an estate $r \in M$ such that $y_{i r} / T_{r} \geqslant \underline{c} / C$ since otherwise $\sum_{s \in M} y_{i s}<\sum_{s \in M} T_{s} \cdot \underline{c} / C$, hence $m\left(C-c_{i}\right)<m C \cdot \underline{c} / C$ and so $C-c_{i}<\underline{c}$, a contradiction. Then

$$
\lambda_{i} \geqslant \frac{y_{i r} u_{i r}}{T_{r}^{2}} \geqslant \frac{\underline{c}}{C} \cdot \frac{u_{i r}}{T_{r}} \geqslant \frac{\underline{c}}{C} \cdot \frac{\underline{u}}{2 C \bar{u}} \cdot \underline{u}=\frac{\underline{u}^{2} \underline{c}}{2 \bar{u} C^{2}} .
$$

Here, the first inequality follows from Lemma 2.3 and the third from part (c).

Existence of a Nash equilibrium, i.e., of a 0-equilibrium, can now be proved by letting $\varepsilon$ approach zero, as follows.

Theorem 2.7 The finite estate problem $(S, \pi)$ has a Nash equilibrium.

Proof. Take a decreasing sequence $0<\varepsilon_{1}, \varepsilon_{2}, \ldots$ converging to 0 with $\varepsilon_{1}<\underline{c}$, and for each $k \in \mathbb{N}$ an $\varepsilon_{k}$-equilibrium $\sigma^{k}$ with associated marginal utilities $\left(\lambda_{i}^{k}\right)_{i \in N}$. We may assume without loss of generality that $\sigma^{k} \rightarrow \sigma$ for some $\sigma \in S$, and in view of Lemma 2.6 we may assume that there are $0<\lambda_{i} \in \mathbb{R}$ such that $\lambda_{i}^{k} \rightarrow \lambda_{i}$ for all $i \in N$. Again by Lemma 2.6, all $T_{s}$ associated with $\sigma$ are positive. The remainder of the proof that $\sigma$ is a Nash equilibrium, is then straightforward by checking the conditions in Lemma 2.3 for $\sigma$ and $\varepsilon=0$. 


\section{The infinite estate problem}

\subsection{The infinite estate problem and game}

The estates are now identified with points in the interval [0,1]. Each agent $i \in N$ has entitlement $c_{i}>0$ and utility function $u_{i}:[0,1] \rightarrow(0, \infty)$ which is piecewise continuous ${ }^{1}$ and positively bounded, i.e., there are $\underline{u}_{i}, \bar{u}_{i} \in \mathbb{R}$ such that $0<\underline{u}_{i} \leqslant u_{i}(s) \leqslant \bar{u}_{i}$ for all $s \in[0,1]$. The pair $(c, u)=\left(\left(c_{i}\right)_{i \in N},\left(u_{i}\right)_{i \in N}\right)$ is an (infinite) estate problem.

Remark 3.1 A solution to such a problem is an $n$-tuple $\left(x_{1}, \ldots, x_{n}\right)$, where for each $i \in N, x_{i}:[0,1] \rightarrow[0,1]$ is a measurable (with respect to the BorelLebesgue measure on $[0,1])$ function such that $\sum_{i \in N} x_{i}(s) \leq 1$ for all $s \in[0,1]$. If $X$ is a measurable subset of $[0,1]$ then agent $i$ derives utility $\int_{X} x_{i}(s) u_{i}(s) d s$ from $X$. The function $x_{i}$ can be interpreted as representing shares allocated to agent $i$, but also as a probability density function: then $\int_{X} x_{i}(s) u_{i}(s) d s$ is the expected utility agent $i$ receives from $X$.

As in the finite case, we consider solutions resulting from Nash equilibria in an associated estate game $(\mathcal{S}, p)$, defined as follows. The players are the agents in $N$, and a strategy of player $i$ is a piecewise continuous nonnegative function $\sigma_{i}$ on $[0,1]$ which satisfies

$$
\int_{0}^{1} \sigma_{i}(s) d s=c_{i} .
$$

The interpretation is that for each (measurable) subset $X \subseteq[0,1], \int_{X} \sigma_{i}$ is the claim put by player $i$ on $X$. The strategy set of player $i$ is denoted by $\mathcal{S}_{i}$, and $\mathcal{S}=\prod_{i \in N} \mathcal{S}_{i}$ is the set of strategy profiles. For a strategy profile $\sigma=\left(\sigma_{j}\right)_{j \in N} \in \mathcal{S}$ and player $i \in N$, we define

$$
\bar{\sigma}_{i}(s)=\left\{\begin{aligned}
\frac{\sigma_{i}(s)}{\sum_{j=1}^{n} \sigma_{j}(s)} & \text { if } \sigma_{i}(s)>0 \\
0 & \text { if } \sigma_{i}(s)=0
\end{aligned}\right.
$$

for all $s \in[0,1]$. Then player $i$ 's payoff from $\sigma$ is

$$
p_{i}(\sigma)=\int_{0}^{1} \bar{\sigma}_{i}(s) u_{i}(s) d s
$$

Definition 3.2 A strategy profile $\sigma \in \mathcal{S}$ is a Nash equilibrium if for all $i \in N$

$$
p_{i}(\sigma) \geqslant p_{i}\left(\sigma \mid \tau_{i}\right)
$$

for all $\tau_{i} \in \mathcal{S}_{i}$, where $\left(\sigma \mid \tau_{i}\right)$ is the strategy profile in which player $i$ plays strategy $\tau_{i}$ while all other players $j$ play $\sigma_{j}$.

${ }^{1}$ I.e., has finitely many points of discontinuity. 
The following lemma gives necessary and sufficient conditions for a strategy profile $\sigma \in \mathcal{S}$ to be a Nash equilibrium of $(\mathcal{S}, p)$. We write $T(s)=\sum_{i \in N} \sigma_{i}(s)$ and $y_{i}(s)=T(s)-\sigma_{i}(s)$ for all $s \in[0,1]$. A proof can be found in the Appendix.

Lemma 3.3 The strategy profile $\sigma \in \mathcal{S}$ is a Nash equilibrium if and only if $T(s)>0$ for almost all $s \in[0,1]$ and for every player $i$ there exists $\lambda_{i}>0$ such that

$$
\lambda_{i} \geqslant \frac{y_{i}(s)}{T(s)^{2}} \cdot u_{i}(s)
$$

for almost all $s \in[0,1]$, with equality if $\sigma_{i}(s)>0 .{ }^{2}$ In the latter case, $\sigma_{i}(s)=$ $T(s)-\lambda_{i} T(s)^{2} / u_{i}(s)$.

\subsection{Connection with the finite estate case}

Let $(M, N, c, u)$ be a finite estate problem. With this problem an infinite estate problem is associated, as follows. For each agent $i \in N$ define the function $\hat{u}_{i}$ on $[0,1]$ by

$$
\hat{u}_{i}(s)= \begin{cases}u_{i k} & \text { if } s \in\left[\frac{k-1}{m}, \frac{k}{m}\right), k=1, \ldots, m \\ u_{i m} & \text { if } s=1\end{cases}
$$

Then $(c, \hat{u})=\left(\left(c_{i}\right)_{i \in N},\left(\hat{u}_{i}\right)_{i \in N}\right)$ is an infinite estate problem. Likewise, a strategy profile $\sigma \in S$ in the finite estate game $(S, \pi)$ associated with $(M, N, c, u)$ can be identified with a strategy profile $\hat{\sigma} \in \mathcal{S}$ in the infinite estate game $(\mathcal{S}, p)$ associated with $(c, \hat{u})$ by definining for each player $i \in N$

$$
\hat{\sigma}_{i}(s)= \begin{cases}\sigma_{i k} & \text { if } s \in\left[\frac{k-1}{m}, \frac{k}{m}\right), k=1, \ldots, m \\ \sigma_{i m} & \text { if } s=1 .\end{cases}
$$

Then, clearly, $p_{i}(\hat{\sigma})=\pi_{i}(\sigma)$ for every player $i \in N$. The games $(S, \pi)$ and $(\mathcal{S}, p)$, however, are essentially different: strategies in $\mathcal{S}_{i}$ do not have to be constant on each of the $m$ intervals in $[0,1]$ corresponding to the $m$ estates of the finite estate game. It turns out, nevertheless, that the Nash equilibria of $(S, \pi)$ and $(\mathcal{S}, p)$ basically coincide.

Theorem 3.4 Let $(M, N, c, u)$ be a finite estate problem, let $(c, \hat{u})$ be the associated infinite estate problem, and let $(S, \pi)$ and $(\mathcal{S}, p)$ be the associated finite and infinite estate games. Then $\tau \in \mathcal{S}$ is a Nash equilibrium in $(\mathcal{S}, p)$ if and only if there is a Nash equilibrium $\sigma \in S$ in $(S, \pi)$ such that $\tau=\hat{\sigma}$ almost everywhere.

Proof. Let $\tau \in \mathcal{S}$ be a Nash equilibrium in $(\mathcal{S}, p)$. For every $k=1, \ldots, m$, we have essentially a homogenous claim game on the interval $X_{k}=[(k-1) / m, k / m]$, as studied in Atlamaz et al. (2008), with entitlements $\int_{X_{k}} \tau_{i}$ for every $i \in N$. Clearly, the restriction of $\tau$ to $X_{k}$ is a Nash equilibrium in this game. By Proposition 6.3 in Atlamaz et al. (2008) it follows that each $\tau_{i}$ is constant almost everywhere on $X_{k}$, with constant value say $\sigma_{i k}$. Then $\sigma$ is a Nash equilibrium in $(S, \pi)$ and $\tau=\hat{\sigma}$ almost everywhere.

\footnotetext{
${ }^{2} \mathrm{~A}$ statement is true for almost all $s \in[0,1]$ if it is true on a subset of $[0,1]$ of measure 1 .
} 
For the converse, let $\tau \in \mathcal{S}$ such that $\tau=\hat{\sigma}$ almost everywhere for some Nash equilibrium $\sigma \in S$ in $(S, \pi)$. Consider player $i \in N$ and a best reply $v_{i} \in \mathcal{S}_{i}$ against $\tau$. For each $k=1 \ldots, m$ consider again the homogenous claim game on $X_{k}$ with entitlements $\int_{X_{k}} \tau_{j}$ for players $j \neq i$ and $\int_{X_{k}} v_{i}$ for player $i$. Again by Proposition 6.3 in Atlamaz et al. (2008) it follows that $v_{i}$ is constant almost everywhere on each $X_{k}$. Since $\sigma$ is a Nash equilibrium in $(S, \pi)$ it follows that for the constant value of $v_{i}$ on $X_{k}$ we can take $\sigma_{i k}$ for each $k=1, \ldots, m$. Thus, $\hat{\sigma}_{i}=\tau_{i}$ is a best reply against $\tau$. Since $i$ was arbitrary, it follows that $\tau$ is a Nash equilibrium in $(\mathcal{S}, p)$.

\subsection{Existence of Nash equilibrium in the infinite estate game}

Existence of a Nash equilibrium in the infinite estate game will be established by approximating this game by finite estate games. The following lemma basically states that for each vector of marginal utilities there can be at most one Nash equilibrium and, moreover, this equilibrium depends continuously on the marginal utilities. See the Appendix for the proof.

Lemma 3.5 Let $(c, u)$ be an infinite estate problem with associated estate game $(\mathcal{S}, p)$, let $s \in[0,1]$, and let $\lambda_{i}>0$ and $\sigma_{i}(s) \geqslant 0, i \in N$, satisfy

$$
\lambda_{i} \geqslant \frac{y_{i}(s)}{T(s)^{2}} \cdot u_{i}(s), \sigma_{i}(s)\left[\lambda_{i}-\frac{y_{i}(s)}{T(s)^{2}} \cdot u_{i}(s)\right]=0 \text { for all } i \in N,
$$

where, as before, $T(s)=\sum_{i \in N} \sigma_{i}(s)$ and $y_{i}(s)=T(s)-\sigma_{i}(s)$. Let $\sigma^{\prime} \in \mathcal{S}$ also satisfy (5). Then $\sigma_{i}^{\prime}(s)=\sigma_{i}(s)$ for all $i \in N$. Moreover, $\sigma(s)$ depends continuously on $\left(\lambda_{i}, u_{i}(s)\right)_{i \in N}$.

We are now sufficiently equipped to prove the existence theorem.

Theorem 3.6 Let $(c, u)$ be an infinite estate problem and let $(\mathcal{S}, p)$ be the associated estate game. Then $(\mathcal{S}, p)$ has a Nash equilibrium.

Proof. For each $k \in \mathbb{N}$ let $M^{k}=\{1, \ldots, k\}$. For each $s \in M^{k}$ and $i \in N$ let $u_{i s}^{k}=u_{i}(s / k)$. Then, for each $k \in \mathbb{N},\left(N, M^{k}, c, u^{k}\right)$ is a finite estate problem. Let $\left(S^{k}, \pi^{k}\right)$ be the associated finite estate game. By Theorem 2.7 this game has a Nash equilibrium $\sigma^{k}$ with associated marginal utilities $\lambda^{k}=\left(\lambda_{1}^{k}, \ldots, \lambda_{n}^{k}\right)$. The finite estate game $\left(S^{k}, \pi^{k}\right)$ can be seen as an infinite (step function) estate game $\left(\mathcal{S}^{k}, p^{k}\right)$ and by Theorem 3.6, this has a Nash equilibrium $\tau^{k}=\hat{\sigma}^{k}$ with the same associated marginal utilities $\lambda^{k}=\left(\lambda_{1}^{k}, \ldots, \lambda_{n}^{k}\right)$. By Lemma 2.6 we may assume that the marginal utilities $\lambda_{i}^{k}$ converge, say $\lambda_{i}^{k} \rightarrow \lambda_{i}>0$ for every $i \in N$. By Lemma 3.5 we can define $\tau_{i}(s)=\lim _{k \rightarrow \infty} \tau_{i}^{k}(s)$ for all $s \in[0,1]$. Since the functions $\tau_{i}$ are bounded by Lemma 2.6, the dominated convergence theorem of Lebesgue implies that each $\tau_{i}$ is integrable and $\int_{0}^{1} \tau_{i}(s) d s=\lim _{k \rightarrow \infty} \int_{0}^{1} \tau_{i}^{k}(s) d s=c_{i}$. Moreover, it is not difficult to prove, using the last claim in Lemma 3.5, that each $\tau_{i}$ is continuous at each point at 
which all utility functions are continuous. Hence, the functions $\tau_{i}$ are piecewise continuous and therefore $\tau \in \mathcal{S}$. Also by Lemma 2.6, $T(s):=\sum_{i \in N} \tau_{i}(s)>0$ for all $s \in[0,1]$. It is straightforward to check the remaining conditions in Lemma 3.3 for $\tau$. Hence, $\tau$ is a Nash equilibrium in $(\mathcal{S}, p)$.

Remark 3.7 A direct proof of Theorem 3.6, i.e., a proof not based on a limiting argument, seems to be complicated by the fact that the set of bounded piecewise continuous functions or even of continuous functions on the interval $[0,1]$ is not compact in any useful sense. This hinders application of a fixed point theorem, such as the Schauder fixed point theorem for infinite dimensional normed vector spaces (cf. Rudin, 1991).

\section{Multiplicity, welfare, and equity properties}

Apart from the nontrivial issue of existence of Nash equilibria in estate games, there are additional questions of interest, to which we provide some answers here. We also indicate some directions for further research.

\subsection{The number of Nash equilibria}

We first work out a relatively simple example, in which we have either one or three Nash equilibria.

Example 4.1 Let $M=\{1,2\}, N=\{1,2\}, u_{11}=u_{22}=1, u_{12}=u_{21}=x>0$, and $c_{1}=c_{2}=1 / 2$. Lemma 2.3 implies the equilibrium conditions

$$
\frac{T_{1}-\sigma_{11}}{T_{1}^{2}}=\frac{T_{2}-\sigma_{12}}{T_{2}^{2}} \cdot x, \frac{\sigma_{11}}{T_{1}^{2}} \cdot x=\frac{\sigma_{12}}{T_{2}^{2}}, T_{1}+T_{2}=2, \sigma_{11}+\sigma_{12}=1 .
$$

Solving this system of four equations in the unknowns $T_{1}, T_{2}, \sigma_{11}$, and $\sigma_{12}$, results in the equation

$$
(x+1)^{2} T^{3}-3(x+1)^{2} T^{2}+2\left(x^{2}+6 x+1\right) T-8 x=0,
$$

where $T=T_{1}$. Since in equilibrium both $T_{1}$ and $T_{2}$ are positive, we are looking for solutions with $0<T<2$. As can be expected, $T=1$ is a solution: this results in the symmetric equilibrium with $T_{1}=T_{2}=1$, namely $\sigma_{1}=$ $(1 /(x+1), x /(x+1))$ and $\sigma_{2}=(x /(x+1), 1 /(x+1))$, as can easily be derived from the four initial conditions. We next factor out $T-1$ and obtain:

$$
\begin{array}{r}
(x+1)^{2} T^{3}-3(x+1)^{2} T^{2}+2\left(x^{2}+6 x+1\right) T-8 x= \\
\quad=(T-1)\left((x+1)^{2} T^{2}-2(x+1)^{2} T+8 x\right)=0 .
\end{array}
$$

This results in the quadratic equation

$$
T^{2}-2 T+\frac{8 x}{(x+1)^{2}}=0
$$


which has solutions

$$
T=1 \pm \sqrt{1-\frac{8 x}{(x+1)^{2}}}
$$

provided

$$
\frac{8 x}{(x+1)^{2}}<1 \Leftrightarrow 0<x^{2}-6 x+1 \Leftrightarrow x \notin[3-\sqrt{8}, 3+\sqrt{8}] .
$$

Hence, for $3-\sqrt{8} \leqslant x \leqslant 3+\sqrt{8}$ the symmetric equilibrium is also the unique equilibrium. For other values of $x$ there are three equilibria: at $x=3 \pm \sqrt{8}$ the equilibrium manifold splits into three branches. For instance, if $x=6$ then $T_{1}=T=1 \pm(1 / 7)$ and $T_{2}=1 \mp(1 / 7)$. For $T_{1}=8 / 7$ the equilibrium is $\sigma=((8 / 35,27 / 35),(32 / 35,3 / 35))$; for $T_{1}=6 / 7$ it is $\sigma=((3 / 35,32 / 35),(27 / 35$, $8 / 35))$. The symmetric equilibrium is $\sigma=((1 / 7,6 / 7),(6 / 7,1 / 7))$.

The next example discusses a case in which the equilibrium is simple and unique. In this example the players have identical preferences.

Example 4.2 Let $v:[0,1] \rightarrow \mathbb{R}$ be a piecewise continuous function such that $\underline{v} \leqslant v(s) \leqslant \bar{v}$ for each $s \in[0,1]$, where $0<\underline{v} \leqslant \bar{v}$. Assume $\int_{0}^{1} v(s) d s=1$. Consider the infinite estate problem $(c, u)$, where each player $i \in N$ has utility function $u_{i}=v .^{3}$ Then the associated infinite estate game $(\mathcal{S}, p)$ has a Nash equilibrium $\sigma^{*}$, given by $\sigma_{i}^{*}(s)=c_{i} v(s)$ for all $s \in[0,1]$. This equilibrium is unique up to a set of measure zero.

To see this, let $\sigma$ be a Nash equilibrium with corresponding marginal utilities $\lambda_{i}, i \in N$ (cf. Lemma 3.3). Denote $C_{i}=C-c_{i}$ for all $i \in N$. Let $i, j \in N$ and suppose without loss of generality that $\lambda_{i} / \lambda_{j} \geqslant C_{i} / C_{j}$. Hence, for all $s \in[0,1]$ with $\sigma_{i}(s)>0$ we have

$$
\frac{C_{j}}{C_{i}} \frac{T(s)-\sigma_{i}(s)}{T(s)^{2}} v(s)=\frac{C_{j}}{C_{i}} \lambda_{i} \geqslant \lambda_{j} \geqslant \frac{T(s)-\sigma_{j}(s)}{T(s)^{2}} v(s)
$$

hence

$$
\frac{C_{j}}{C_{i}}\left(T(s)-\sigma_{i}(s)\right) \geqslant T(s)-\sigma_{j}(s) .
$$

Therefore

$$
C_{j}=\frac{C_{j}}{C_{i}} \cdot C_{i}=\frac{C_{j}}{C_{i}} \int_{0}^{1}\left(T(s)-\sigma_{i}(s)\right) d s \geqslant \int_{0}^{1}\left(T(s)-\sigma_{j}(s)\right) d s=C_{j}
$$

which implies that (6) holds with equality almost everywhere. Since $i$ and $j$ were arbitrary, we conclude that $\sigma(s)=\left(\sigma_{1}(s), \ldots, \sigma_{n}(s)\right)$ is a solution of the system

$$
\frac{C_{1}}{C_{i}}\left(T(s)-\sigma_{i}(s)\right)=T(s)-\sigma_{1}(s), \quad i \in N
$$

\footnotetext{
${ }^{3}$ Or a positive multiple of $v$, that is irrelevant.
} 
for almost all $s$. System (7) is a homogenous system of linear equations and so every multiple of a solution is again a solution. Therefore, we can normalize solutions by setting $T(s)-\sigma_{1}(s)=1$ (clearly, $T(s)-\sigma_{1}(s) \neq 0$ ). Then, using matrix notation, (7) can be written as an non-homogenous system

$$
\left[\begin{array}{ccccc}
0 & 1 & 1 & \cdots & 1 \\
1 & 0 & 1 & \cdots & 1 \\
1 & 1 & 0 & \cdots & 1 \\
\vdots & \vdots & \vdots & \ddots & 1 \\
1 & 1 & 1 & \cdots & 0
\end{array}\right]\left[\begin{array}{c}
\sigma_{1}(s) \\
\sigma_{2}(s) \\
\sigma_{3}(s) \\
\vdots \\
\sigma_{n}(s)
\end{array}\right]=\left[\begin{array}{c}
1 \\
C_{2} / C_{1} \\
C_{3} / C_{1} \\
\vdots \\
C_{n} / C_{1}
\end{array}\right] .
$$

The coefficient matrix is invertible and so the system has a unique solution. It is easy to check that $\sigma(s)=c / C_{1}$ is this solution. Hence, any Nash equilibrium must be proportional to $c$, and thus $\sigma^{*}$ is the unique Nash equilibrium (up to a set of measure zero).

A noteworthy further observation is that in this unique Nash equilibrium $\sigma^{*}$ each player plays a max-min strategy, i.e., a strategy that maximizes the minimal payoff, where the minimum is taken over all strategy combinations of the opponents. To see this, note that for any $\sigma \in \mathcal{S}$ we have

$$
\sum_{i \in N} \int_{0}^{1} \bar{\sigma}_{i}(t) v(t) d t=\int_{0}^{1} \sum_{i \in N} \bar{\sigma}_{i}(t) v(t) d t=1
$$

i.e., the sum of the payoffs of the players is constant and equal to 1. Suppose player 1 plays $\sigma_{1}^{*}$. Following the logic above, the other players maximize their joint payoff $\sum_{i=2}^{n} \int_{0}^{1} \bar{\sigma}_{i}(t) v(t) d t=\int_{0}^{1} \sum_{i=2}^{n} \bar{\sigma}_{i}(t) v(t) d t$ from the strategy profile $\left(\sigma_{1}^{*}, \sigma_{2}, \ldots, \sigma_{n}\right)$ by choosing $\left(\sigma_{2}, \ldots, \sigma_{n}\right)$ such that $\sum_{i=2}^{n} \sigma_{i}(t)=\left(\sum_{i=2}^{n} c_{i}\right) v(t)$ for each $t \in[0,1]$. This implies that $\sigma_{1}^{*}$ guarantees a payoff of $\int_{0}^{1}\left(c_{1} / C\right) v(t) d t$ to player 1 . In the same way, each player $i \in N$ can guarantee $\int_{0}^{1}\left(c_{i} / C\right) v(t) d t$ by playing $\sigma_{i}^{*}$. Since the sum of all these payoffs is equal to 1 , each $\sigma_{i}^{*}$ is a max-min strategy.

A general investigation of the cardinality of the set of Nash equilibria is left for further research.

\subsection{Welfare properties of Nash equilibria}

How do Nash equilibria of the (in)finite estate game perform in terms of welfare? For the homogenous case studied in Atlamaz et al. (2008) this is not so much an issue: preferences (utilities) over the estates are homogenous, there is basically only one estate and each player is indifferent between different parts of the estates and only cares about the share of the estate that he obtains. Every Nash equilibrium is therefore Pareto optimal. In the present model, where preferences are no longer homogenous and players care not only about the quantity but also the quality of the estate, this is of course different. Nevertheless, we will show that Nash equilibria cannot Pareto dominate each other. 
Let $(c, u)$ be an infinite estate problem with associated game $(\mathcal{S}, p)$. Consider two different Nash equilibria $\sigma$ and $\sigma^{\prime}$, with marginal utility vectors $\lambda$ and $\lambda^{\prime}$. Without loss of generality we assume that the conditions in Lemma 3.3 are satisfied for all $s \in[0,1]$. Recall that $\bar{\sigma}(s)$ and $\bar{\sigma}^{\prime}(s)$ denote the vectors of shares of the players at each estate $s \in[0,1]$.

Lemma 4.3 Let $i, j \in N$ with $\lambda_{i}^{\prime} / \lambda_{i} \geqslant \lambda_{j}^{\prime} / \lambda_{j}$ and let $s \in[0,1]$. Suppose $\bar{\sigma}_{j}(s)>0$ and $\bar{\sigma}_{i}^{\prime}(s)>\bar{\sigma}_{i}(s)$. Then $\bar{\sigma}_{j}^{\prime}(s)>\bar{\sigma}_{j}(s)$.

Proof. By the conditions in Lemma 3.3 we obtain

$$
\frac{\lambda_{j}}{u_{j}(s)}=\frac{T(s)-\sigma_{j}(s)}{T(s)^{2}}=\frac{1}{T(s)}\left(1-\bar{\sigma}_{j}(s)\right)
$$

and similarly

$$
\frac{\lambda_{j}^{\prime}}{u_{j}(s)} \geqslant \frac{1}{T^{\prime}(s)}\left(1-\bar{\sigma}_{j}^{\prime}(s)\right)
$$

so that

$$
\frac{\lambda_{j}^{\prime}}{\lambda_{j}} \geqslant \frac{T(s)}{T^{\prime}(s)} \frac{\left(1-\bar{\sigma}_{j}^{\prime}(s)\right)}{\left(1-\bar{\sigma}_{j}(s)\right)} .
$$

Similarly, using $\bar{\sigma}_{i}^{\prime}(s)>0$, we obtain

$$
\frac{\lambda_{i}^{\prime}}{\lambda_{i}} \leqslant \frac{T(s)}{T^{\prime}(s)} \frac{\left(1-\bar{\sigma}_{i}^{\prime}(s)\right)}{\left(1-\bar{\sigma}_{i}(s)\right)} .
$$

Hence

$$
\frac{\left(1-\bar{\sigma}_{j}^{\prime}(s)\right)}{\left(1-\bar{\sigma}_{j}(s)\right)} \leqslant \frac{\left(1-\bar{\sigma}_{i}^{\prime}(s)\right)}{\left(1-\bar{\sigma}_{i}(s)\right)}<1,
$$

which implies $\bar{\sigma}_{j}^{\prime}(s)>\bar{\sigma}_{j}(s)$.

We will use Lemma 4.3 to prove the already announced result that one Nash equilibrium cannot Pareto dominate another one. Specifically, we have the following result.

Theorem 4.4 Let $(c, u)$ be an infinite estate problem with associated game $(\mathcal{S}, p)$ and let $\sigma$ and $\sigma^{\prime}$ be Nash equilibria such that $\bar{\sigma} \neq \bar{\sigma}^{\prime}$ on a subset of $[0,1]$ of positive measure. Then there are $j, k \in N$ with $p_{j}(\sigma)<p_{j}\left(\sigma^{\prime}\right)$ and $p_{k}(\sigma)>p_{k}\left(\sigma^{\prime}\right)$.

Proof. Let $O$ be the set of players $i$ with $\bar{\sigma}_{i}(s)=\bar{\sigma}_{i}^{\prime}(s)$ almost everywhere and let $\bar{N}=N \backslash O$. Clearly, $\bar{N}$ contains at least two players. Take player $j \in \bar{N}$ with minimal ratio $\lambda_{j}^{\prime} / \lambda_{j}$. For almost all $s \in[0,1]$ we have $\sum_{i \in \bar{N}} \bar{\sigma}_{i}(s)=\sum_{i \in \bar{N}} \bar{\sigma}_{i}^{\prime}(s)$; so for almost all $s$, if $\bar{\sigma}_{j}(s) \neq \bar{\sigma}_{j}^{\prime}(s)$ then there is a player $i \in \bar{N}$ with $\bar{\sigma}_{i}(s)<$ $\bar{\sigma}_{i}^{\prime}(s)$. But then Lemma 4.3 implies that $\bar{\sigma}_{j}(s)<\bar{\sigma}_{j}^{\prime}(s)$. Hence, for almost all $s, \bar{\sigma}_{j}(s) \leqslant \bar{\sigma}_{j}^{\prime}(s)$, with strict inequality on a subset of $[0,1]$ of positive measure. Thus, $p_{j}(\sigma)<p_{j}\left(\sigma^{\prime}\right)$.

Now let $k \in \bar{N}$ be a player with maximal ratio $\lambda_{k}^{\prime} / \lambda_{k}$. Then player $k$ has minimal ratio $\lambda_{k} / \lambda_{k}^{\prime}$ and by an argument analogous to the preceding one we obtain $p_{k}(\sigma)>p_{k}\left(\sigma^{\prime}\right)$. 
Example 4.5 For the three Nash equilibria in Example 4.1 where we took $x=$ 6 , the payoffs are $(5.6,4.9)$ for the equilibrium $((3 / 35,27 / 35),(32 / 35,3 / 35))$; $(4.9,5.6)$ for the equilibrium $((3 / 35,32 / 35),(27 / 35,8 / 35))$; and approximately $(5.29,5.29)$ for the symmetric equilibrium $((1 / 7,6 / 7),(6 / 7,1 / 7))$. The payoff pair $(6,6)$, obtained by taking $\sigma_{1}=(0,1)$ and $\sigma_{2}=(1,0)$, is Pareto optimal in the game $(\mathcal{S}, p)$, and it is not difficult to show that there is no payoff pair with sum of payoffs more than 12 .

Example 4.5 illustrates the fact that generally speaking Nash equilibrium payoffs are not Pareto optimal. An interesting question (which we leave for further research) is how well Nash equilibria perform in terms of welfare. For instance, one can ask how big the loss of welfare is compared to a Pareto optimal division of the estates. ${ }^{4}$

\subsection{Envy-freeness}

Envy-freeness is an appealing criterion in order to judge whether an allocation is fair or equitable. If, however, the agents in an estate problem have different entitlements then it is difficult to compare their allocations in a solution to the problem. Generally speaking, larger entitlements lead to larger shares of the estates in any reasonable solution, and so an agent with a relatively small entitlement will envy an agent with a large entitlement. Therefore, we restrict attention to problems in which agents have equal entitlements. For simplicity we only consider finite estate problems.

Let $(M, N, c, u)$ be a finite estate problem with $c_{i}=c_{j}$ for all $i, j \in N$ and with associated estate game $(S, \pi)$ and let $\sigma \in S$ such that $\sum_{i \in N} \sigma_{i s}>0$ for all $s \in M$. We say that $\sigma$ is envy-free if for all $i, j \in N$ :

$$
\sum_{s \in M} \frac{\sigma_{i s}}{\sum_{k \in N} \sigma_{k s}} \cdot u_{i s} \geqslant \sum_{s \in M} \frac{\sigma_{j s}}{\sum_{k \in N} \sigma_{k s}} \cdot u_{i s} .
$$

This means that each player $i$ weakly prefers his own allocation to that of another player $j$. If the opposite strict inequality holds then we say that player $i$ envies player $j$. The following example shows that Nash equilibria need not be envy-free.

Example 4.6 Let $N=\{1,2,3\}, M=\{1,2\}, c_{1}=c_{2}=c_{3}=1 / 2$ and $u_{11}=$ $u_{22}=10, u_{12}=u_{21}=u_{31}=1, u_{32}=16 / 25$. Then

$$
\sigma_{11}=\sigma_{22}=1, \sigma_{31}=\frac{2}{3}, \sigma_{32}=\frac{1}{3}
$$

is a Nash equilibrium, and player 3 envies player 1 .

The next result shows that for two players every Nash equilibrium is envyfree.

\footnotetext{
${ }^{4}$ I.e., what is the 'price of anarchy', cf. Johari and Tsitsiklis, 2004.
} 
Theorem 4.7 Let $(M,\{1,2\}, c, u)$ be a finite estate problem with $c_{1}=c_{2}$, and let $\sigma$ be a Nash equilibrium in the associated game $(S, \pi)$. Then $\sigma$ is envy-free.

Proof. We shall prove that

$$
\sum_{s \in M} \frac{\sigma_{2 s}-\sigma_{1 s}}{T_{s}} \cdot u_{1 s} \leqslant 0
$$

which means that player 1 does not envy player 2. Since $\sigma_{i s}>0$ for $i=$ 1,2 and for all $s \in M$, we have $\left(y_{i s} / T_{s}^{2}\right) \cdot u_{i s}=\left(y_{i 1} / T_{1}^{2}\right) \cdot u_{i 1}$, hence $u_{i s}=$ $\left(T_{s}^{2} / y_{i s}\right)\left(y_{i 1} / T_{1}^{2}\right) \cdot u_{i 1}$, for $i=1,2$ and $s \in M$. Then

$$
\sum_{s \in M} \frac{\sigma_{2 s}-\sigma_{1 s}}{T_{s}} \cdot u_{1 s}=\frac{\sigma_{21}-\sigma_{11}}{T_{1}} \cdot u_{11}+\sum_{s \in M \backslash\{1\}} \frac{\sigma_{2 s}-\sigma_{1 s}}{T_{s}} \frac{T_{s}^{2}}{y_{1 s}} \frac{y_{11}}{T_{1}^{2}} \cdot u_{11} .
$$

Since we just want show that the sum is negative, we can multiply by the positive number $T_{1}^{2} /\left(y_{11} \cdot u_{11}\right)$. This yields

$$
\sum_{s \in M}\left(\sigma_{2 s}-\sigma_{1 s}\right) \frac{T_{s}}{y_{1 s}} .
$$

Now, since $y_{1 s}=\sigma_{2 s}$ and $\sigma_{1 s}+\sigma_{2 s}=T_{s}$, we have

$\sum_{s \in M}\left(\sigma_{2 s}-\sigma_{1 s}\right) \frac{T_{s}}{y_{1 s}}=\sum_{s \in M}\left(\sigma_{2 s}-\sigma_{1 s}\right) \frac{T_{s}}{\sigma_{2 s}}=\sum_{s \in M} \frac{2 \sigma_{2 s}-T_{s}}{\sigma_{2 s}} T_{s}=\sum_{s \in M} 2 T_{s}-\frac{T_{s}^{2}}{\sigma_{2 s}}$.

For any given $\left(T_{s}\right)_{s \in M}$ the $\sigma^{\prime}$ that maximizes the expression on the righthand side under the constraint $\sum_{s \in M} \sigma_{2 s}^{\prime}=m \cdot c_{2}$ satisfies the property that $T_{s} / \sigma_{2 s}^{\prime}$ is constant for all $s$, as can easily be checked using Lagrange. Since $\sum_{s \in M} T_{s} / \sum_{s \in M} \sigma_{2 s}^{\prime}=m\left(c_{1}+c_{2}\right) / m c_{2}=2 m c_{2} / m c_{2}=2$, we have $T_{s} / \sigma_{2 s}^{\prime}=2$ for all $s$. Hence

$$
\sum_{s \in M} 2 T_{s}-\frac{T_{s}^{2}}{\sigma_{2 s}} \leqslant \sum_{s \in M} 2 T_{s}-\frac{T_{s}}{\sigma_{2 s}^{\prime}} T_{s}=\sum_{s \in M} 2 T_{s}-2 T_{s}=0 .
$$

Hence player 1 does not envy player 2. Similarly one shows that player 2 does not envy player 1 .

In the proof of this theorem it used that in a Nash equilibrium every estate is claimed by at least two players, so that in a two-player estate game every player puts a positive claim on every estate in an equilibrium. If there are more than two players then even if every player puts a positive claim on every estate in a Nash equilibrium, this does not imply that such an equilibrium is envy-free, as the next example shows.

Example 4.8 Let $N=\{1,2,3\}, M=\{1,2\}, c_{1}=c_{2}=c_{3}=6, u_{i 1}=1$ for $i=1,2,3, u_{12}=16 / 35, u_{22}=48 / 25, u_{32}=16 / 25$. Then

$$
\sigma_{11}=\sigma_{22}=5, \sigma_{12}=\sigma_{21}=1, \sigma_{31}=4, \sigma_{32}=2
$$

is a Nash equilibrium, and player 3 envies player 1 . 
If the entitlements of the agents in an estate problem are not equal, then it is not obvious how to define envy-freeness. Further exploration of this subject is left for future research.

\section{A Appendix}

Proof of Lemma 2.3. For the only-if part, let $\sigma \in S^{\varepsilon}$ be an $\varepsilon$-equilibrium. If $\varepsilon>0$ then $T_{s} \geqslant n \varepsilon>0$ for each $s \in M$; if $\varepsilon=0$ then $T_{s}=0$ for some $s$ would imply that each player can improve by putting a small claim on $s$, a contradiction. Consider player $i \in N$, then $\sigma_{i}$ solves the problem

$$
\max _{\tau_{i} \in S_{i}} \sum_{s \in M} \frac{\tau_{i s} u_{i s}}{y_{i s}+\tau_{i s}} \text { subject to } \sum_{s \in M} \tau_{i s}=m c_{i} \text { and } \tau_{i s} \geqslant \varepsilon \text { for all } s \in M .
$$

Considering the Lagrangian for this problem with multiplier $\lambda_{i}$ for the constraint $\sum_{s \in M} \tau_{i s}=m c_{i}$, it follows from the Kuhn-Tucker conditions that

$$
\lambda_{i} \geqslant \frac{d}{d \tau_{i s}}\left(\frac{\tau_{i s} u_{i s}}{y_{i s}+\tau_{i s}}\right)_{\mid \tau_{i s}=\sigma_{i s}}=\frac{y_{i s} u_{i s}}{\left(y_{i s}+\sigma_{i s}\right)^{2}}=\frac{y_{i s} u_{i s}}{T_{s}^{2}} \text { for all } s \in M
$$

with equality if $\sigma_{i s}>\varepsilon$. It is easy to see that $\lambda_{i}$ must be positive: otherwise, $y_{i s}=0$ for all $s$, which means that for all $j \neq i$ we would have $\sum_{s \in M} \sigma_{j s}=0$, a plain contradiction.

For the if-part, it is sufficient to observe that for every player $i$, under the conditions stated, $\sigma_{i}$ is a stationary point of the Lagrangian with multiplier $\lambda_{i}$ and that the Lagrangian is concave.

Proof of Lemma 3.3. For the only-if part, let $\sigma$ be a Nash equilibrium. If $T(s)=0$ for all $s \in X$ where $X$ would have measure larger than zero, then an arbitrary player could improve by putting a very small positive claim on $X$. Consider player $i$, then $\sigma_{i}$ solves the maximization problem

$$
\max _{\tau_{i}} \int_{0}^{1} \frac{\tau_{i}(s)}{y_{i}(s)+\tau_{i}(s)} u_{i}(s) d s \quad \text { subject to } \quad \int_{0}^{1} \tau_{i}(s) d s=c_{i} .
$$

This maximization problem can be formulated as an optimal control problem

$$
\begin{aligned}
\max _{x} \int_{0}^{1} \frac{x(s) u_{i}(s)}{y_{i}(s)+x(s)} d s & \quad \dot{X}(s)=-x(s), x(s) \geqslant 0, X(0)=c_{i}, X(1)=0
\end{aligned}
$$

with piecewise continuous control variable $x(s)$. The corresponding Lagrangian is

$$
L=\frac{x(s) u_{i}(s)}{y_{i}(s)+x(s)}-\pi(s) x(s)+\mu(s) x(s)
$$

and the necessary conditions for an optimum are

$$
\frac{\partial L}{\partial x(s)}=0, \frac{\partial L}{\partial \pi(s)}=\dot{X}(s), \frac{\partial L}{\partial X(s)}=-\dot{\pi}(s), \mu(s) x(s)=0, \mu(s) \geqslant 0, x(s) \geqslant 0 .
$$


Since $\partial L / \partial X(s)=0$, there is a $\lambda_{i}$ such that $\pi(s)=\lambda_{i}$ for all $s \in[0,1]$. Then, $\partial L / \partial x(s)=0$ implies

$$
\frac{y_{i}(s) u_{i}(s)}{\left(y_{i}(s)+x(s)\right)^{2}}-\lambda_{i}+\mu(s)=0 \text { for all } s \in[0,1] .
$$

Hence, if $x(s)>0$ then $\mu(s)=0$ and $\lambda_{i}=\frac{y_{i}(s) u_{i}(s)}{\left(y_{i}(s)+x(s)\right)^{2}}$, and if $x(s)=0$ then $\mu(s) \geqslant 0$ and $\lambda_{i} \geqslant \frac{y_{i}(s) u_{i}(s)}{\left(y_{i}(s)+x(s)\right)^{2}}$. Furthermore, $x(s) \geqslant 0$ for all $s$ and $\int_{0}^{1} x(s) d s=c_{i}$ since $\dot{X}(s)=-x(s)$ for all $s$. Hence, all these conditions are satisfied for $\sigma_{i}$. Clearly, $\lambda_{i}$ must be positive.

For the if-part, we provide the following direct argument. Let the stated conditions hold for $\sigma \in \mathcal{S}$, let $i \in N$ and $\sigma^{\prime} \in \mathcal{S}_{i}$. We show that player $i$ 's payoff from $\sigma^{\prime}$ is not larger than his payoff from $\sigma$, i.e., that

$$
\int_{0}^{1} \frac{\sigma_{i}^{\prime}(s)}{y_{i}(s)+\sigma_{i}^{\prime}(s)} \cdot u_{i}(s) d s \leqslant \int_{0}^{1} \frac{\sigma_{i}(s)}{y_{i}(s)+\sigma_{i}(s)} \cdot u_{i}(s) d s .
$$

Without loss of generality we have assumed here that the denominators in both integrals are positive for every $s \in[0,1]$ : for the right-hand side the denominator is positive almost everywhere by assumption, and for the left-hand side we may approximate $\sigma^{\prime}$ arbitrarily close by a strategy of player $i$ that is positive everywhere. Now

$$
\begin{aligned}
& \int_{0}^{1} \frac{\sigma_{i}^{\prime}(s)}{y_{i}(s)+\sigma_{i}^{\prime}(s)} \cdot u_{i}(s) d s-\int_{0}^{1} \frac{\sigma_{i}(s)}{y_{i}(s)+\sigma_{i}(s)} \cdot u_{i}(s) d s \\
= & \int_{0}^{1} \frac{\left(\sigma_{i}^{\prime}(s)-\sigma_{i}(s)\right) y_{i}(s)}{\left(y_{i}(s)+\sigma_{i}^{\prime}(s)\right)\left(y_{i}(s)+\sigma_{i}(s)\right)} \cdot u_{i}(s) d s \\
= & \int_{0}^{1}\left(\sigma_{i}^{\prime}(s)-\sigma_{i}(s)\right) \frac{y_{i}(s)+\sigma_{i}(s)}{y_{i}(s)+\sigma_{i}^{\prime}(s)} \frac{y_{i}(s)}{\left(y_{i}(s)+\sigma_{i}(s)\right)^{2}} \cdot u_{i}(s) d s \\
\leqslant & \int_{0}^{1}\left(\sigma_{i}^{\prime}(s)-\sigma_{i}(s)\right) \frac{y_{i}(s)+\sigma_{i}(s)}{y_{i}(s)+\sigma_{i}^{\prime}(s)} \lambda_{i} d s .
\end{aligned}
$$

For all $s \in[0,1]$ we have

$$
\sigma_{i}^{\prime}(s)-\sigma_{i}(s)>0 \Longleftrightarrow \frac{y_{i}(s)+\sigma_{i}(s)}{y_{i}(s)+\sigma_{i}^{\prime}(s)}<1
$$

hence

$$
\left(\sigma_{i}^{\prime}(s)-\sigma_{i}(s)\right) \frac{y_{i}(s)+\sigma_{i}(s)}{y_{i}(s)+\sigma_{i}^{\prime}(s)} \leqslant \sigma_{i}^{\prime}(s)-\sigma_{i}(s) .
$$


It follows that

$$
\begin{aligned}
\int_{0}^{1}\left(\sigma_{i}^{\prime}(s)-\sigma_{i}(s)\right) \frac{y_{i}(s)+\sigma_{i}(s)}{y_{i}(s)+\sigma_{i}^{\prime}(s)} \lambda_{i} d s & \leqslant \int_{0}^{1}\left(\sigma_{i}^{\prime}(s)-\sigma_{i}(s)\right) \lambda_{i} d s \\
& =\left(c_{i}-c_{i}\right) \lambda_{i}=0
\end{aligned}
$$

which completes the proof of the if-part.

The last claim in the lemma, $\sigma_{i}(s)=T(s)-\lambda_{i} T(s)^{2} / u_{i}(s)$ if $\sigma_{i}(s)>0$, is straightforward.

The following lemma is an auxiliary result used in the proof of Lemma 3.5 below.

Lemma A.1 Let $\left(a_{1}, \ldots, a_{n}\right)$ be a vector of positive numbers and let $\left(a_{(1)}, \ldots\right.$, $\left.a_{(n)}\right)$ denote a permutation of these numbers such that $a_{(1)} \leqslant \ldots \leqslant a_{(n)}$. Then

(i) There is a unique $j \in\{1, \ldots, n\}$ such that

$$
(j-1) a_{(j)}<\sum_{i=1}^{j} a_{(i)} \leqslant(j-1) a_{(j+1)},
$$

where $a_{(n+1)}=\infty$. If $j^{*}=j^{*}\left(a_{1}, \ldots, a_{n}\right)$ is this unique value, then $j^{*} \geqslant 2$.

(ii) The function $T:\left(a_{1}, \ldots, a_{n}\right) \mapsto\left(j^{*}-1\right) / \sum_{i=1}^{j^{*}} a_{(i)}$ with $j^{*}=j^{*}\left(a_{1}, \ldots\right.$, $\left.a_{n}\right)$, is continuous.

Proof of Lemma A.1. (i) First note that for $j=1$ the inequalities in (9) imply $0<a_{(i)} \leqslant 0$, an impossibility. Suppose that (9) does not hold for any $j \in\{2, \ldots, n-1\}$. We claim that (9) holds for $j=n$. If not, then $(n-1) a_{(n)} \geqslant$ $\sum_{i=1}^{n} a_{(i)}$. This implies $(n-2) a_{(n)} \geqslant \sum_{i=1}^{n-1} a_{(i)}$, hence $(n-2) a_{(n-1)} \geqslant \sum_{i=1}^{n-1} a_{(i)}$ since otherwise $j=n-1$ would satisfy (9). Repeating this argument, we find $a_{(2)} \geqslant a_{(1)}+a_{(2)}$, a contradiction. Hence, (9) holds for some $j \in\{2, \ldots, n\}$. Let $j^{*} \geq 2$ be the minimal $j$ for which (9) holds. By the second inequality in (9) we have $\left(j^{*}-1\right) a_{\left(j^{*}+1\right)} \geqslant \sum_{i=1}^{j^{*}} a_{(i)}$ and thus $j^{*} a_{\left(j^{*}+1\right)} \geqslant \sum_{i=1}^{j^{*}+1} a_{(i)}$. This implies that (9) does not hold for $j^{*}+1$ instead of $j^{*}$. Also, for $k \geqslant 2$,

$$
\left(j^{*}+k-1\right) a_{\left(j^{*}+k\right)} \geqslant j^{*} a_{\left(j^{*}+1\right)}+a_{\left(j^{*}+2\right)}+\ldots+a_{\left(j^{*}+k\right)} \geqslant \sum_{i=1}^{j^{*}+k} a_{(i)} .
$$

So (9) does not hold for any $j^{*}+k, k \geqslant 2$.

(ii) Clearly, if both inequalities in (9) are strict for $j^{*}$, then $T$ is continuous at $\left(a_{1}, \ldots, a_{n}\right)$. If the second inequality is an equality, then it is sufficient to prove that the value of $T$ does not change if we replace $j^{*}$ by $j^{*}+1$, i.e., that

$$
\left(j^{*}-1\right) / \sum_{i=1}^{j^{*}} a_{(i)}=j^{*} / \sum_{i=1}^{j^{*}+1} a_{(i)}
$$


To show this, write $T\left(j^{*}\right)=\left(j^{*}-1\right) / \sum_{i=1}^{j^{*}} a_{(i)}$ and note that

$$
\begin{aligned}
\frac{1}{j^{*}} \sum_{i=1}^{j^{*}+1} a_{(i)} & =\frac{\left(j^{*}-1\right) \frac{1}{j^{*}-1} \sum_{i=1}^{j^{*}} a_{(i)}+a_{\left(j^{*}+1\right)}}{j^{*}} \\
& =\frac{\left(j^{*}-1\right) \frac{1}{T\left(j^{*}\right)}+\frac{1}{T\left(j^{*}\right)}}{j^{*}}=\frac{1}{T\left(j^{*}\right)}
\end{aligned}
$$

implying (10).

Proof of Lemma 3.5. For convenience we drop $s$ from the notations in this proof. We write $a_{i}=\lambda_{i} / u_{i}$ for every $i$ and without loss of generality assume that $a_{1} \leqslant \ldots \leqslant a_{n}$. Let $I=\left\{i \in N \mid \sigma_{i}>0\right\}$. The proof proceeds by two claims.

Claim 1: Let $j \in I$ and $k<j$. Then $k \in I$.

To prove this claim, note that $\lambda_{j}=y_{j} u_{j} / T^{2}<T u_{j} / T^{2}=u_{j} / T$, so $\lambda_{j} / u_{j}<$ $1 / T$. Hence $y_{k} / T_{k}^{2} \leqslant \lambda_{k} / u_{k}=a_{k} \leqslant a_{j}=\lambda_{j} / u_{j}<1 / T$. This implies $y_{k}<T$ and, thus, $\sigma_{k}>0$, i.e., $k \in I$.

Claim 1 implies that $I=\{1, \ldots, j\}$ for some $j \in N$.

Claim 2: Let $I=\{1, \ldots, j\}$. Then

$$
a_{j}<\frac{1}{j-1} \sum_{i=1}^{j} a_{i} \leqslant a_{j+1} .
$$

To prove this, first note that $\sigma_{i}=T-\lambda_{i} T^{2} / u_{i}=T-a_{i} T^{2}$ for all $i \in I$. This implies $T=\sum_{i \in I} \sigma_{i}=j T-\sum_{i \in I} a_{i} T^{2}$, and thus $1 / T=[1 /(j-1)] \sum_{i=1}^{j} a_{i}$. Then

$$
a_{j}=\frac{y_{j}}{T^{2}}<\frac{T}{T^{2}}=\frac{1}{T}=\frac{1}{j-1} \sum_{i=1}^{j} a_{i},
$$

and

$$
a_{j+1} \geqslant \frac{y_{j+1}}{T^{2}}=\frac{T}{T^{2}}=\frac{1}{T}=\frac{1}{j-1} \sum_{i=1}^{j} a_{i} .
$$

This completes the proof of Claim 2. The first part of the lemma now follows from part (i) of Lemma A.1. By part (ii) of Lemma A.1, $T(s)$ depends continuously on $\left(\lambda_{i}, u_{i}(s)\right)_{i \in N}$, and therefore $\sigma(s)$ depends continuously on $\left(\lambda_{i}, u_{i}(s)\right)_{i \in N}$ as well.

\section{References}

Atlamaz, M., C. Berden, H. Peters, and D. Vermeulen (2008): "Non-cooperative solutions for claims problems," METEOR Research Memorandum 08/038, Maastricht. 
Aumann, R. and M. Maschler (1985): "Game Theoretic Analysis of a Bankruptcy Problem from the Talmud," Journal of Economic Theory, 36, 195-213.

Berliant, M. (1985): "An Equilibrium Existence Result for an Economy with Land," Journal of Mathematical Economics, 14, 53-56.

Berliant, M., W. Thomson and K. Dunz (1992): "On the Fair Division of a Heterogeneous Commodity," Journal of Mathematical Economics, 21, 201-206.

Brams, S., and A.D. Taylor (1996): From Cake-Cutting to Dispute Resolution. Cambridge University Press, Cambridge, UK.

Cramton, P., R. Gibbons, and P. Klemperer (1987): "Dissolving a Partnership Efficiently," Econometrica, 55, 615-632.

Gairing, M., T. Lücking, M. Mavronicolas, and B. Monien (2009): "Computing Nash Equilibria for Scheduling on Restricted Parallel Links," working paper, ICSI, Berkeley, USA

Hotelling, H. (1929): "Stability in Competition," The Economic Journal, 39, $41-57$.

Johari, R., and J.N. Tsitsiklis (2004): "Efficiency Loss in a Network Resource Allocation Game," Mathematics of Operations Research, 29, 407-435.

Merolla, J., M. Munger, and M. Tofias (2003): "Lotto, Blotto, or Frontrunner: An Analysis of Spending Patterns by the National Parties Committees in the 2000 Presidential Election," mimeo, Duke University.

O'Neill, B. (1982): "A Problem of Rights Arbitration from the Talmud," Mathematical Social Sciences, 2, 345-371.

Rudin, W. (1991): Functional Analysis. McGraw-Hill Science.

Thomson, W. (2003): "Axiomatic and Game-theoretic Analysis of Bankruptcy and Taxation Problems: a survey," Mathematical Social Sciences, 45, 249297. 